\title{
Electrical Properties of Newly Calcified Tissues on the Surface of Silver Ion Administrated Hydroxyapatite Scaffolds
}

\author{
Manjushree M. Bahir ${ }^{1 *}$, Rajendra S. Khairnar², Megha P. Mahabole ${ }^{2 *}$ \\ ${ }^{1}$ National Center for Cell Science, Pune, India \\ ${ }^{2}$ Swami Ramanand Teerth Marathwada University, Nanded, India \\ Email: ^manjub@nccs.res.in, ^mpmstrmunsps@gmail.com
}

How to cite this paper: Bahir, M.M., Khairnar, R.S. and Mahabole, M.P. (2020) Electrical Properties of Newly Calcified Tissues on the Surface of Silver Ion Administrated Hydroxyapatite Scaffolds. Journal of Biomaterials and Nanobiotechnology, $11,83-100$.

https://doi.org/10.4236/jbnb.2020.112006

Received: November 18, 2019

Accepted: February 1, 2020

Published: February 4, 2020

Copyright $\odot 2020$ by author(s) and Scientific Research Publishing Inc. This work is licensed under the Creative Commons Attribution International License (CC BY 4.0).

http://creativecommons.org/licenses/by/4.0/

(c) (i) Open Access

\begin{abstract}
The application of electric field to graft materials has significant contribution in bone healing mechanism. Hence, the aim of this study is to develop conductive hydroxyapatite (HAp) scaffolds by introducing different concentrations of silver ion into its structure and demonstrate its impact on in vitro bioactivity and electrical properties. Hydroxyapatite was synthesized by wet chemical method and calcium ions from HAp structure have been partially replaced by silver ions. The HAp and Ag-HAp nanocomposites were characterized by Fourier-transform infrared, Raman spectroscopy, XRD and EDAX for functional group and phase formation analysis as well as to confirm existence of silver ions in HAp structure respectively. Bioactivity of these scaffolds was assessed by using simulated body fluid. The surface morphology, structural analysis and electrical properties of scaffolds before and after formation of newly calcified tissues on its surface were examined via scanning electron microscopy (SEM), XRD, FTIR, dielectric and impedance spectroscopy techniques. Overall, our finding suggests that the administration of silver ions in HAp scaffold boosts bioactivity and has strong correlation with electrical properties.
\end{abstract}

\section{Keywords}

Hydroxyapatite, Wet Chemical Method, Bioactivity, Electrical Properties

\section{Introduction}

Bone loss is major concern worldwide due to several reasons such as accidents, bone diseases like osteoporosis, bone tumor as a consequence bone turns to be fracture. Though minor fracture can be healed by themselves by causing in- 
flammatory reaction at fracture site, major bone defect needs to endure surgical treatment by grafting biocompatible material at fracture region. Hydroxyapatite $\left[\mathrm{Ca}_{10}\left(\mathrm{PO}_{4}\right)_{6}(\mathrm{OH})_{2}\right.$, abbreviated as HAp], being bioactive and biocompatible, can be an exciting material in emerging field of synthetic bone graft due to its chemical similarity with the mineral part of the human bone [1] [2] [3]. In the biomedical field, HAp has been explored for biosensor, bioimaging, drug delivery, protein purification, gene delivery and other biomedical applications [4] [5] [6]. Also, it is reported that the efficacy of HAp in terms of its physical and biological properties could be enhanced by doing advancement into its structure [7] [8]. Hence, the immense research has been explored in modification of HAp structure by substituting metal ion species like copper $\left(\mathrm{Cu}^{2+}\right)$, manganese $\left(\mathrm{Mn}^{2+}\right)$, silicon $\left(\mathrm{Si}^{4+}\right)$, iron $\left(\mathrm{Fe}^{3+}\right)$, strontium $\left(\mathrm{Sr}^{2+}\right)$, cobalt $\left(\mathrm{CO}^{2+}\right)$, zinc $\left(\mathrm{Zn}^{2+}\right)$, silver $\left(\mathrm{Ag}^{+}\right)$ for improvement in its biological premises for specific applications in biomedical field [8]-[15]. However, among various trace metal ions, introduction of $\mathrm{Ag}^{+}$ ion in HAp can have an advantage of excellent biocompatibility, satisfactory stability, antibacterial nature and noncytotoxicity and this can help to prevent post transplantation infection risk of implant material [16] [17]. The inclusion of silver ions in HAp structure promotes bioactivity and such coating on metal offers corrosion resistance [18] [19] [20]. Therefore, silver particles are widely used in various medical applications like bone prostheses, artificial teeth, and bone coating [21] [22]. Furthermore, the cation exchange rate of HAp is found to be very high with silver ions.

Recent advances in orthopedic therapy have shown that application of electrical field to bone stimulates bone growth, promotes osseointegration, and boosts bone density [23] [24] [25] [26]. Further, few studies demonstrated that accumulation of electrical charges on HAp graft plays a significant role in osteoconduction mechanism and in reconstruction of bone occupancy at damaged part of bone after implantation [27] [28] [29]. Also, Maharbiz et al. have observed that impedance measurements can be used to monitor early stage bone healing process and recovery of bone fracture [30]. Similarly, Tian et al. detected the variations in HAp electrical impedance value due to structural changes during bone regeneration process and illustrated that impedance spectroscopy can hold considerable potential for quantitative bone healing analysis [31]. The dielectric properties of HAp, Sr-HAp and Mn-HAp incubated in simulated body fluid as a function of incubation period have been studied by our group [9] [32] [33]. These studies have shown that electrical properties, specifically dielectric properties of biomaterials are of great interest to understand bone healing mechanism.

Hence, by considering the coalition between the electrical properties and bone healing process, efforts have been made to investigate the in vitro electrical property of newly calcified tissue on silver ion incorporated HAp scaffold. Emphasis has also been given to correlate the dielectric, photoluminescence properties of Ag-HAp with the bioactivity. 


\section{Methods}

\subsection{Hydroxyapatite Modification}

Nano crystalline hydroxyapatite (HAp) bioceramic was synthesized by wet-chemical precipitation method [32]. Further, HAp structure was modified by partial replacement of calcium ions by silver ions $\left(\mathrm{Ag}^{+}\right)$via ion exchange process, carried out at room temperature. Synthesized Ca-HAp and Ag-HAp nano-bioceramic materials were characterized by XRD, FTIR, SEM/EDAX, Raman spectroscopy and BET techniques. The Ag-HAp samples with variable silver concentrations (0.001 M, $0.005 \mathrm{M}$, and $0.025 \mathrm{M}$ ) were uniaxially pressed at 5-ton pressure to form compact disc shaped scaffolds of $13 \mathrm{~mm}$ diameter and $2 \mathrm{~mm}$ thickness. The prepared scaffolds were then heat treated at $500^{\circ} \mathrm{C}$ for $2 \mathrm{~h}$ and used as scaffolds to study further in vitro bioactivity and electrical properties.

\subsection{Preparation of Simulated Body Fluid (SBF) and In Vitro Bioactivity}

In vitro bioactivity study of HAp and Ag-HAp scaffolds was carried out using pseudo body fluid (SBF) in static mode condition. The pseudo body fluid was prepared by dissolving, one by one, appropriate amount of various chemicals viz. $\mathrm{NaCl}, \mathrm{NaHCO}_{3}, \mathrm{KCl}, \mathrm{Na}_{2} \mathrm{HPO}_{4} \cdot 2 \mathrm{H}_{2} \mathrm{O}, \mathrm{MgCl}_{2} \cdot 6 \mathrm{H}_{2} \mathrm{O}, \mathrm{CaCl}_{2} \cdot 2 \mathrm{H}_{2} \mathrm{O}, \mathrm{Na}_{2} \mathrm{SO}_{4}$ in double distilled water so as to have molar concentrations of various inorganic ions, $\mathrm{pH}$, and temperature similar to natural body fluid [34]. Ag-HAp scaffolds with variable silver ion concentrations were incubated in freshly prepared SBF for fixed time duration. Similarly, Ag-HAp scaffolds with fixed silver concentration $(0.005 \mathrm{M})$ were placed in SBF for variable time durations for detail dielectric studies. At the end of predetermined incubation duration, the scaffolds were removed from the SBF solution and rinsed with distilled water. Further, these scaffolds were dried at room temperature and used for analysis.

\subsection{Dielectric and Electrical Characterization}

The dielectric measurements such as dielectric constant, dissipation factor, and impedance were carried out using Quad Tech make LCR 7600 meter at room temperature in frequency range of $10 \mathrm{~Hz}$ to $1 \mathrm{MHz}$ for already prepared scaffolds.

\subsection{Characterization}

The structural analysis was carried out using Rigaku to make X-ray diffractometer with $\mathrm{CuKa}$ radiation $(\mathrm{K}=1.543 \AA)$. FTIR and Raman spectroscopic techniques were used for identification and conformation of the functional groups. Fourier transform infrared (FTIR) spectra of scaffolds were recorded in 400 to $4000 \mathrm{~cm}^{-1}$ range with a resolution of $4 \mathrm{~cm}^{-1}$ by using Shimadzu make spectrophotometer. Raman spectra of scaffolds were analyzed in the spectral range of 100 to $1100 \mathrm{~cm}^{-1}$ with the spectral resolution of $4 \mathrm{~cm}^{-1}$ by using Raman microscope (Horiba 800, France). Surface morphology and elemental analysis of Ag-HAp scaffolds before and after SBF treatment were examined by scanning 
electron microscope (SEM) (with LiecaStereoscan 440 model SEM) coupled with Energy dispersive X-ray analyzer (EDAX). Further, surface area and porosity of these scaffolds were decided by Bruaauer-Emmett-Teller (BET) method (Supplementary information). The PL spectra for Ag-HAp scaffolds were conducted, before and after SBF incubation, at room temperature using Horiba FL3-22-1186C-2609 $\left(\lambda_{\text {exc }}=415 \mathrm{~nm}\right)$.

\section{Results and Discussion}

\subsection{XRD Analysis}

The XRD profiles of Ag-HAp samples as a function concentration is presented in Figure 1(a). The profiles of $\mathrm{Ag}^{+}$containing hydroxyapatite samples resemble

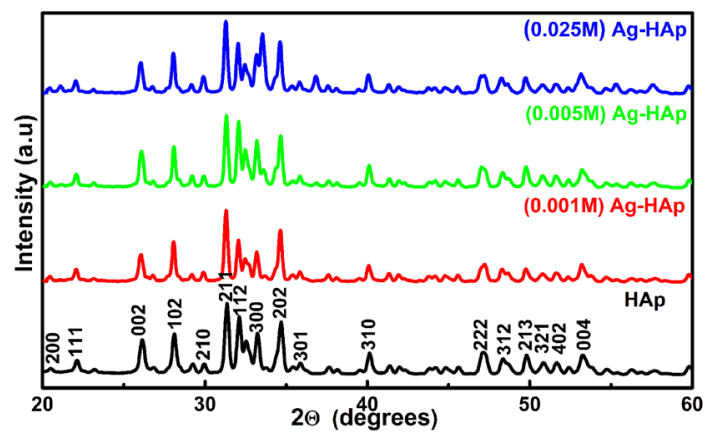

(a)

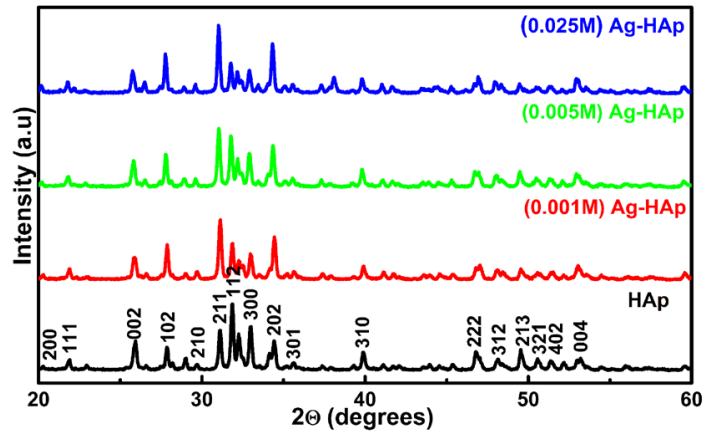

(b)

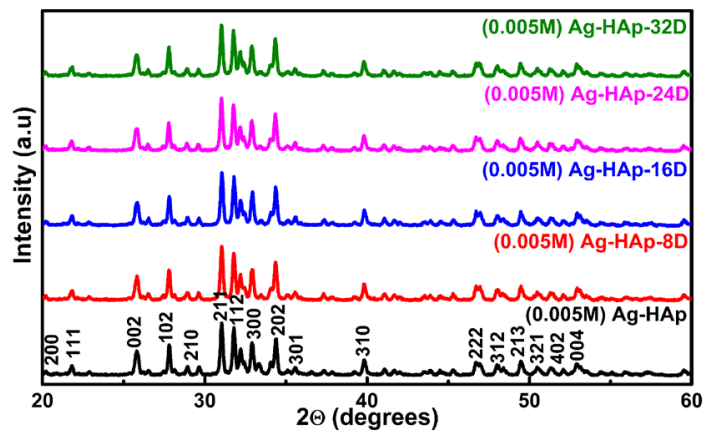

(c)

Figure 1. XRD spectra of Ag-HAp Scaffolds: (a) as a function of $\mathrm{Ag}^{+}$concentration; (b) for 8 days of SBF treatment; and (c) after SBF treatment for variable time durations ranging from 8 - 32 days. 
with XRD pattern of the parent Ca-HAp (JCPDS 09-0432) wherein major characteristic apatite peaks are found to be present. This not only indicates the development of hexagonal hydroxyapatite phase but also confirms that ionic substitution does not change the apatite structure. No evidence is found for any other phases. It is also observed from Figure 1(a) that due to the incorporation of silver ions in hydroxyapatite matrix, intensities of almost all peaks decrease compared to Ca-HAp. In particular the intensity of peak assigned to (211) plane decreases with increase in silver concentration. The XRD spectra of Ag-HAp samples after incubation in SBF are presented in Figure 1(b) \& Figure 1(c). The XRD of all incubated samples are found to be essentially indistinguishable and matches well with that of Ca-HAp as provided in JCPDS 09-0432.

It is observed that hydroxyapatite structure is retained by all samples during incubation in SBF. This also indicates that no other ions, such as sodium, ammonium, potassium, magnesium, chloride and nitrate, are involved into HAp structure after incubation in SBF. The absence of such ions in HAp may be attributed to the formation of calcium and phosphate rich apatite layer on surface of Ag-HAp scaffolds.

\subsection{FTIR Analysis}

The FTIR spectra of ion exchanged hydroxyapatite nano-bioceramics, in the range of $500-1300 \mathrm{~cm}^{-1}$, are presented in Figure 2(a) and the spectrum of pristine HAp (before incubation) is considered as a reference. The FTIR spectra of pristine Ca-HAp and Ag-HAp samples as a function of ion concentration, prior to immersion in SBF, clearly demonstrate the presence of characteristic apatite absorption peaks corresponding to the various vibrational modes of phosphate and hydroxyl groups. The absorption peaks appearing near 1090 and $1030 \mathrm{~cm}^{-1}$ are because of $v_{3}$ fundamental stretching vibrations of $\left(\mathrm{PO}_{4}^{3-}\right)$ functional group [35]. The bands appearing at 967 and $941 \mathrm{~cm}^{-1}$ are attributed to $v_{1}$ symmetric stretching mode of $\left(\mathrm{PO}_{4}^{3-}\right)$ [36]. The absorption band near $632 \mathrm{~cm}^{-1}$ corresponds to hydroxyl liberation mode. The absorption bands due to $v_{4}$ fundamental bending mode of $\left(\mathrm{PO}_{4}^{3-}\right)$ group are present near $543 \mathrm{~cm}^{-1}$ and $603 \mathrm{~cm}^{-1}$ [35] [36]. It is found that the absorption increases with increase in ion concentration. Hence, it can be concluded that higher the ion concentration, higher is the absorption.

Typical FTIR spectra of incubated HAp samples, showing change in absorption peak intensities of various groups, are presented in Figure 2(b) \& Figure 2(c). The FTIR spectra of the Ca-HAp and Ag-HAp scaffolds after incubation, for all incubation durations (Figure 2(c)) \& for fixed incubation period (Figure 2(b)), demonstrate presence of phosphate and hydroxyl functional groups. The most appreciable change is observed for the phosphate peak centered at 1030 $\mathrm{cm}^{-1}$ compared to other peaks. A new distinct phosphate group band has appeared near to $1120 \mathrm{~cm}^{-1}$. The change in absorption intensities corresponding to the phosphate and hydroxyl groups, after immersion in SBF for fixed \& variable 


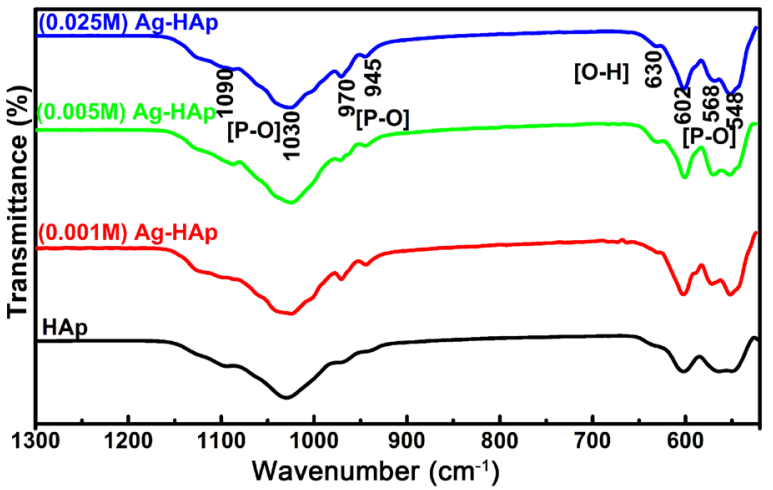

(a)

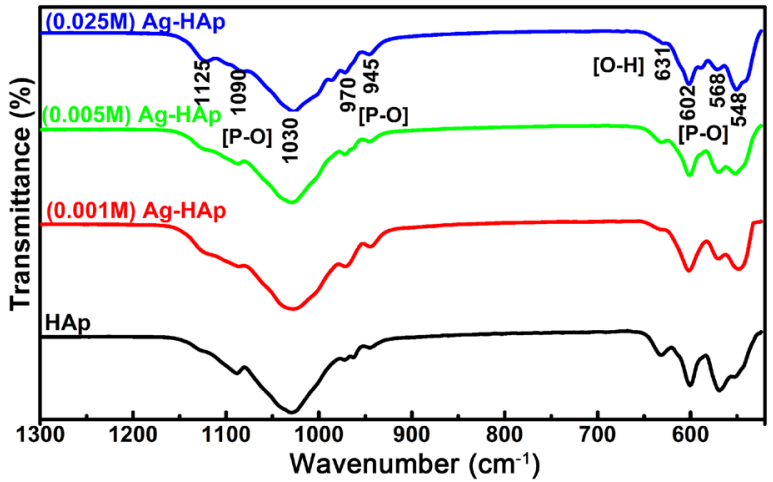

(b)

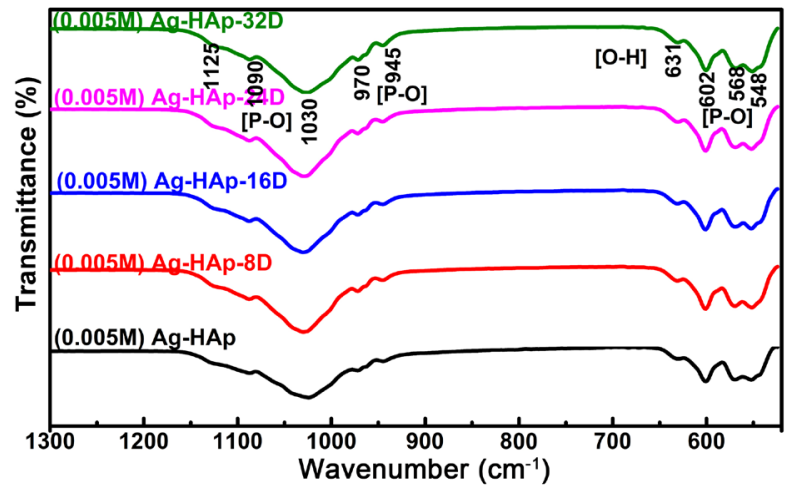

(c)

Figure 2. FTIR spectra of Ag-HAp scaffolds: (a) with variation in $\mathrm{Ag}^{+}$concentration; (b) for 8 days of incubation in SBF; and (c) upon SBF treatment for 8 - 32 days time durations.

durations, and the appearance of absorption peak near $1120 \mathrm{~cm}^{-1}$ for $\mathrm{HPO}_{4}^{2+}$ confirms the apatite growth.

The Raman spectra of SBF incubated Ag-HAp scaffolds (0.005 M), provided in supplementary data, also show presence of characteristic phosphate modes at $\sim 429,705$ and $958 \mathrm{~cm}^{-1}$ which can be assigned to hydroxyapatite vibration modes. Almost all Ag-HAp scaffolds, prior to \& upon incubation, manifest highest intensity vibrational mode at $\sim 958 \mathrm{~cm}^{-1}$ with shoulders at 943 and $970 \mathrm{~cm}^{-1}$. This strong Raman peak at $\sim 958 \mathrm{~cm}^{-1}$ provides complementary information in 
tune with FTIR and clearly corroborates growth of apatite on scaffold surface during incubation.

\subsection{SEM Analysis}

Figure 3(a) represents the surface morphology of Ca-HAp and Ag-HAp scaffolds as a function of ionic concentration. The surface of Ca-HAp scaffold exhibits the presence of a large number of grains but of very small size. In addition, small pores are observed on the surface. The surface of Ag-HAp scaffold with lowest silver concentration shows larger size grains whereas Ag-HAp scaffold with highest silver concentration possesses highest porosity. The corresponding EDAX spectra show the increase in atomic weight percent of silver with increase in ion concentrations ensuring incorporation of silver ions in Ca-HAp scaffolds.

The SEM micrographs of Ca-HAp and Ag-HAp scaffolds, after incubation for 8 days, are presented in Figure 3(b). Presence of small size spherical grains on the surface Ca-HAp scaffold compared to Ag-HAp scaffolds reveals slow growth
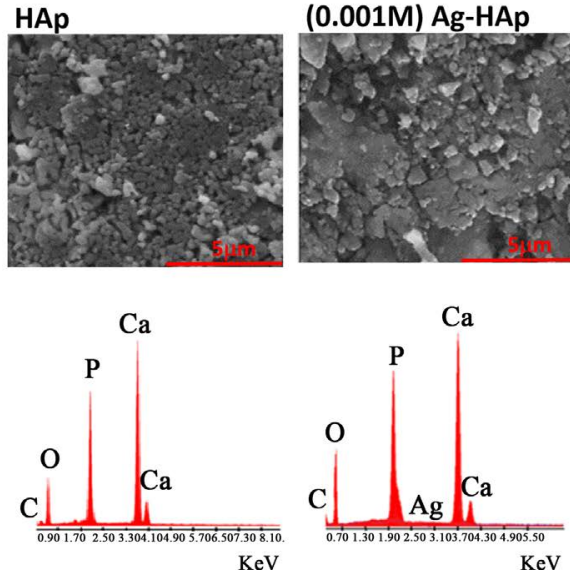
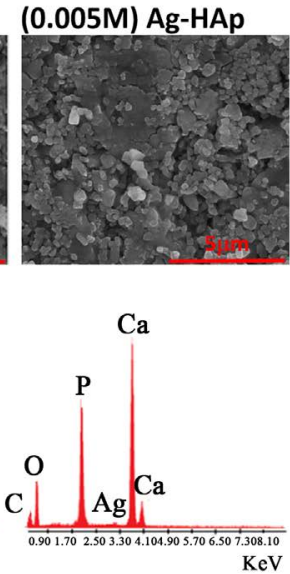
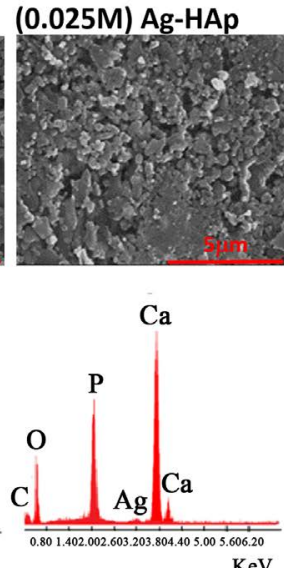

(a)

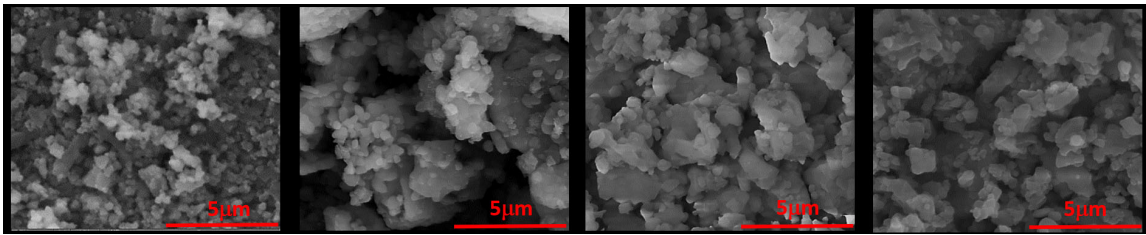

(b)

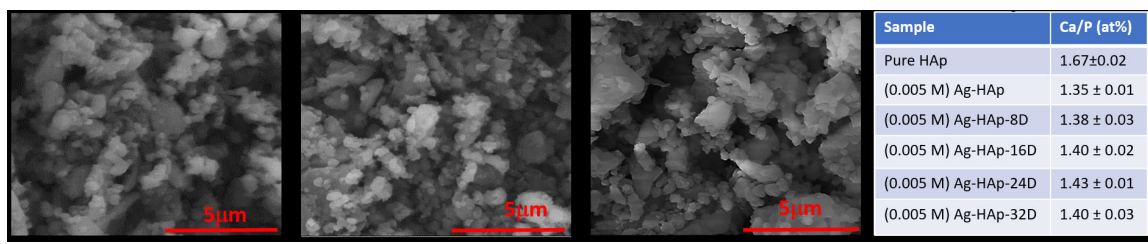

(c)

Figure 3. (a) SEM images and corresponding EDAX spectra of Ag-HAp scaffolds prior to incubation in SBF as a function of $\mathrm{Ag}^{+}$concentration; (b) SEM images after SBF treatment for 8 days; and (c) SEM images after SBF treatment for variable time of immersion and EDAX analysis. 
rate. The surfaces of Ag-HAp scaffolds are found to be fully covered with newly formed calcified tissues wherein size of new formed grains scaffold surface observed to be increasing with silver ion contents. This indicates dominance of higher growth rates and coalescence phenomenon with silver ion concentration. It is also observed that for fixed silver ion loading and variable incubation periods, agglomeration phenomenon plays important role for higher incubation period (Figure 3(c)).

Further, the EDAX data for incubated Ag-HAp samples reveals that $\mathrm{Ca} / \mathrm{P}$ ratio reaches to a maximum value of 1.43 after 24 days. These results show that Ag-HAp nano-ceramic scaffolds have ability to act as templates for apatite layer formation on their surfaces and can be considered as superior bioactive nano-ceramics than Ca-HAp.

Since the process of apatite layer formation takes place at the bioactive HAp scaffold surface/SBF solution interface, it depends on majorly on surface ions. Due to presence of hydroxyl $\left(\mathrm{OH}^{-}\right)$and phosphate $\left(\mathrm{PO}_{4}^{3-}\right)$ ions on the HAp surface, the HAp surface carries negative charge. These negative ions attract the positive calcium ions from the SBF solution and result in the formation of Ca-rich surface acquiring positive charge. This positively charged surface then interacts with negatively charged phosphate ions from SBF fluid and formation of Ca-poor HAp layer takes place on the surface. This is followed by the transformation of Ca-poor HAp layer to crystalline, stoichiometric HAp via crystallization process during further incubation period.

\subsection{Dielectric Studies}

\subsubsection{Dielectric Constant}

Figure 4(a) presents the change in dielectric constant for Ag-HAp scaffolds with change in frequency of an applied ac field as a function of concentration and the plots are compared with that for Ca-HAp. All the scaffolds show similar behavior of decrease in dielectric constant with increase in frequency. The dielectric constant of Ca-HAp drastically changes after addition of silver ions into HAp structure. The dielectric constants for Ag-HAp scaffolds are found to be smaller than that for Ca-HAp as depicted in Figure 4(a). It is observed that as the silver ion content increases, dielectric constant decreases. The dielectric constant for higher silver ion concentration $(0.025 \mathrm{M})$ is observed to the smallest (approximately near to 8 ) in comparison with other silver concentrations. The study clearly reveals the dependence of dielectric constant on ionic concentration.

The effect of incubation on dielectric constant of Ag-HAp as a function of frequency of applied ac field is depicted in Figure 4(b) \& Figure 4(c). It can be concluded that dielectric constant changes upon SBF treatment. The dielectric constant of Ag-HAp scaffold ( $0.005 \mathrm{M})$ enhances from 24 (prior to incubation) to $\approx 58$ when incubated for 8 days. The typical trend of decrease in dielectric constant with increase in incubation period can be visualized from Figure 4(c). It is well known that the dielectric properties of hydroxyapatite are mainly due to the motions of $\mathrm{OH}^{-}$ions with applied ac field and inclusion of silver ions may 


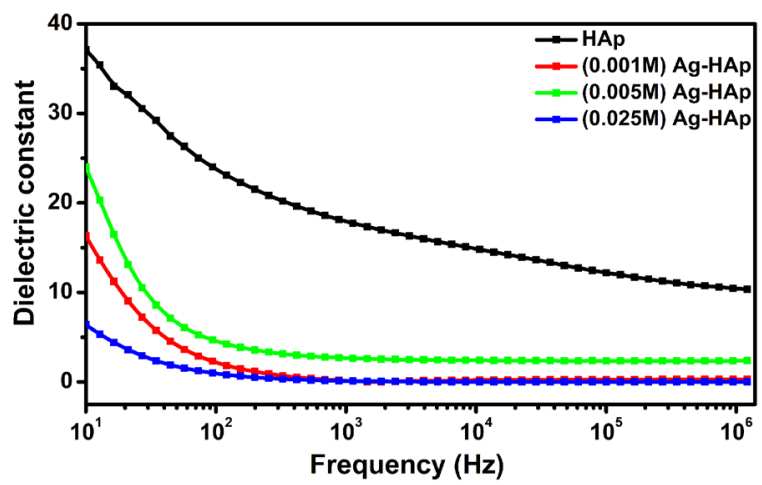

(a)

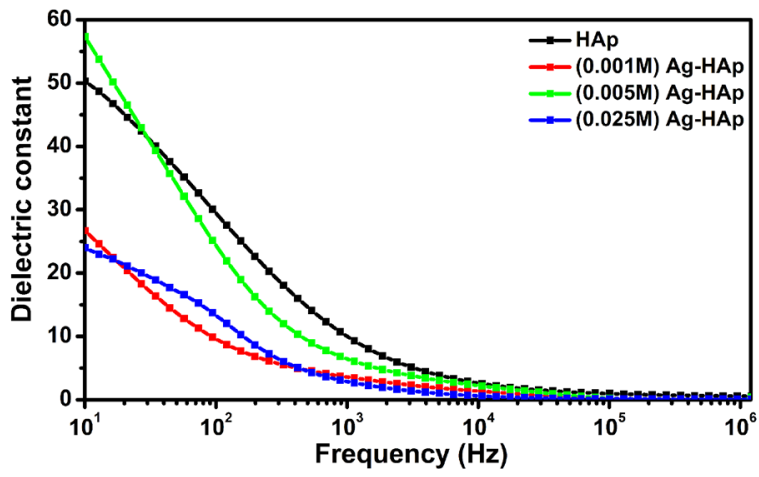

(b)

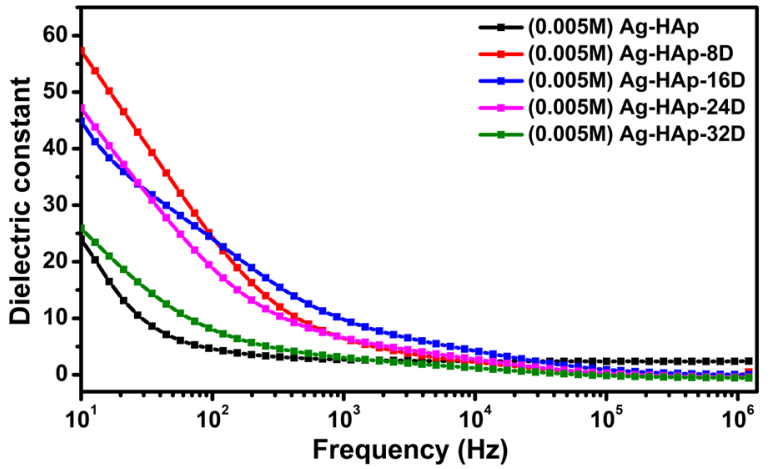

(c)

Figure 4. Dielectric constant as a function of frequency for Ag-HAp nano-ceramic scaffolds: (a) before incubation; (b) after incubation for fixed duration for variable silver ion content; and (c) after incubation for variable time duration for fixed silver ion content.

be responsible for change of electrical dipoles of $\mathrm{OH}^{-}$ions leading to the decrease in dielectric permittivity of Ag-HAp.

The dielectric properties of Ag-HAp, before and after incubation, display high dielectric permittivity at low frequencies which falls off with increase in frequency reaching a constant value for all samples. High values of dielectric constants at low frequencies are obvious because the hydroxyapatite belongs to bio-ceramic category and the behavior of dielectric permittivity is related to free dipoles oscillating in an applied alternating field. At very low alternating field frequencies, electric dipoles specifically due to hydroxyl ions follow the field al- 
terations and contribute to high value of dielectric constant at lower frequency.

With increase in frequency of ac field, the dipoles start lagging behind the field reversal leading to slight decrease in dielectric constant followed by abrupt drop in dielectric constant. At still higher frequencies, the ions cannot follow the alternating field. As a result, polarization decreases and capacitor offers low reactance to the sinusoidal signal minimizing the conduction losses in the resistor and hence, dielectric constant decreases. The high dielectric constant for lower incubation period at lower frequencies may be due to the fact that the free charges buildup at interfaces within bulk of the scaffold (interfacial Maxwell-Wagner polarization) indicating apatite layer formation.

\subsubsection{Dielectric Loss}

Typical variation in dielectric loss with frequency for Ag-HAp scaffolds, prior to incubation, is presented in Figure 5(a). The Ag-HAp scaffolds demonstrate similar trend wherein dielectric loss initially decreases with increase in frequency in the lower frequency region followed by sudden increase with a hump occurring near about $100 \mathrm{~Hz}$. Further increase in frequency leads to decrease in loss. The position of this maximum remains unchanged, but height of loss maxima decreases with increase in silver concentration. This indicates that height of loss maxima depends on silver ion concentration.

The dielectric loss for incubated HAp scaffolds, for fixed \& variable incubation periods, as a function of frequency of applied ac field is presented in Figure 5(b) and Figure 5(c). The incubated Ag-HAp scaffolds show same behavior with a hump occurring near about $100 \mathrm{~Hz}$. However, in case of incubated samples, the position and value of these maxima (height) are observed to be the function of immersion time. As the SBF treatment period increases, position of peak shifts to lower frequency. In case of 32 days of incubation, frequency corresponding maxima coinciding with that for prior to incubation. Moreover, it is observed that height of dielectric loss decreases with increase in period of incubation. Shift in frequencies corresponding to maxima as a function of incubation period leads to change in relaxation time. The sharp peaks in dielectric loss spectra, in the lower frequency region, can be attributed to ionic conduction relaxation. The fast decreasing trend of dissipation factor at low frequency may be due to the presence of high ionic conductivity in Ag-HAp scaffolds. A relative decrease in magnitude of loss with increase in incubation period is an indication of weakening of the dipolar ordering in the material at that frequency.

The Debye relaxation theory states that the loss peak appears when the alternating field is in phase with dielectrics since the condition of $\tau \omega=1$ (where $\omega=$ $2 \pi f \& \tau$ is relaxation time) is satisfied.

\subsubsection{Cole-Cole Plot}

Cole-Cole plots for Ag-HAp scaffolds are presented in Figures 6(a)-(c). The horizontal axis is the resistance $(Z)$ i.e. the real part of the impedance and vertical axis is the reactance $\left(Z^{\prime}\right)$ i.e. imaginary part of the impedance. The presence 


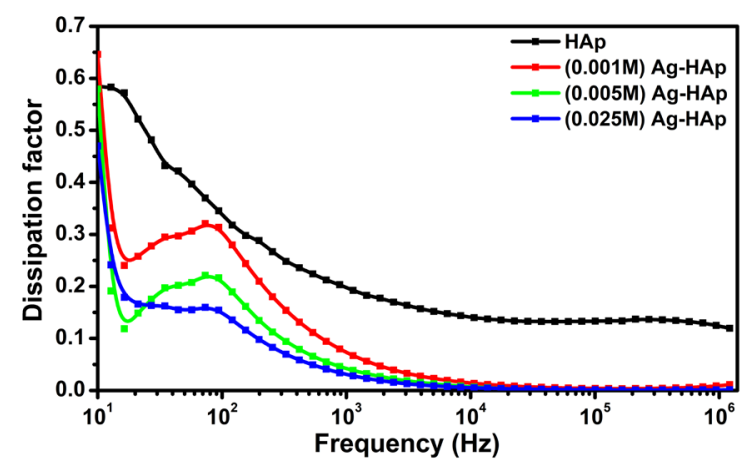

(a)

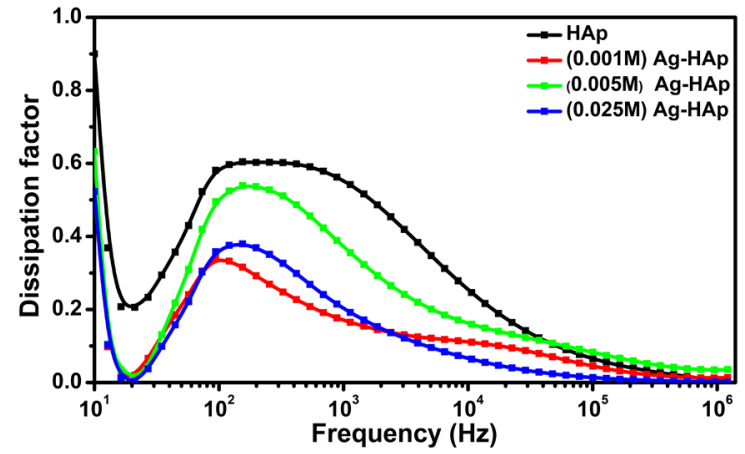

(b)

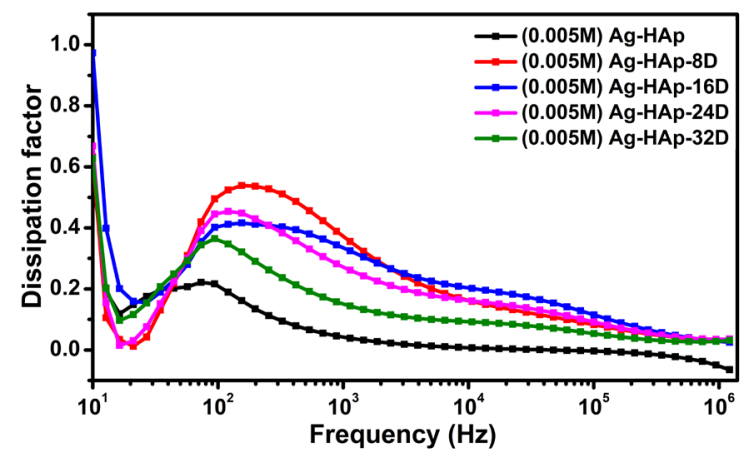

(c)

Figure 5. Dissipation factor as a function of frequency for Ag-HAp scaffolds: (a) with variation in silver ion content prior to incubation; (b) after incubation in SBF for fixed duration (8 days) for variable silver ion content; and (c) after incubation for variable time duration for fixed silver ion content $(0.005 \mathrm{M})$.

of two semicircles for all scaffolds reveals the poly-dispersed nature of the material and represents two RC elements. The low frequency arcs are due to grain boundaries and higher frequency arcs indicate grain effect. The intercepts of low frequency arc \& high frequency arc on the real axis designate grain boundary resistance $\left(R_{g b}\right)$ and bulk resistance $\left(R_{g}\right)$ respectively. The values of $R_{g}, \tau$ and $R_{g b}$ are listed in Table 1 . Similarly, grain capacitance $\left(C_{g}\right)$, grain boundary capacitance $\left(C_{g b}\right)$ values are calculated from following equation:

$$
C_{g} / C_{g b}=\frac{1}{R_{g} / R_{g b} \times 2 \pi f_{c}}
$$




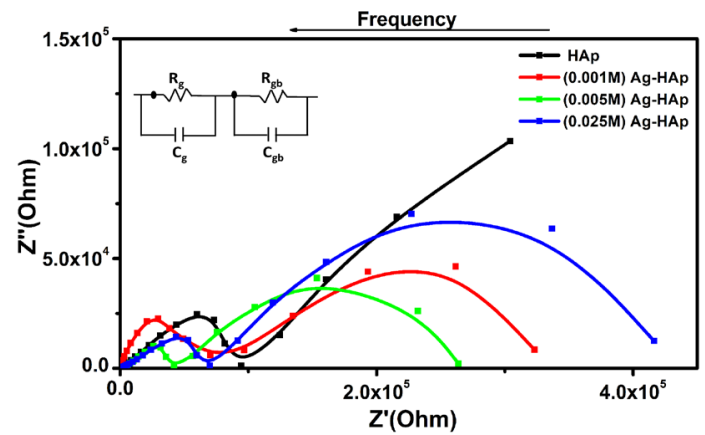

(a)

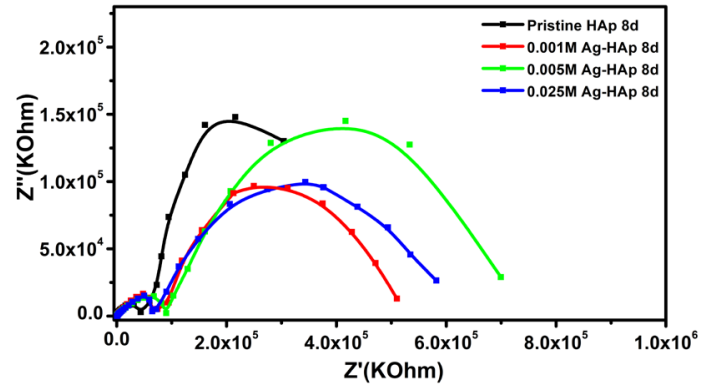

(b)

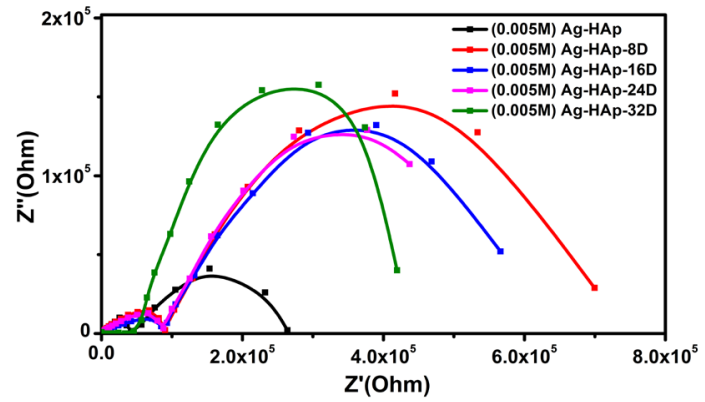

(c)

Figure 6. Impedance spectra of Ag-HAp scaffolds: (a) before incubation as a function of silver ion loading; (b) after incubation for 8 days for variable silver ion contents; and (c) after incubation for variable time duration for fixed silver ion content $(0.005 \mathrm{M})$.

Table 1. Bulk resistance $\left(R_{g}\right)$, relaxation time $(\tau)$ and grain boundary resistance $\left(R_{g b}\right)$ derived from impedance spectra of Ca-HAp and Ag-HAp scaffolds before and after incubation in SBF.

\begin{tabular}{|c|c|c|c|c|c|c|}
\hline \multirow[b]{2}{*}{ Scaffolds } & \multicolumn{3}{|c|}{ Before incubation in SBF } & \multicolumn{3}{|c|}{ After incubation in SBF for 8 days } \\
\hline & $\begin{array}{l}\text { Bulk } \\
\text { resistance } \\
\quad(\mathrm{Rg})\end{array}$ & $\begin{array}{c}\text { Grain } \\
\text { boundary } \\
\text { resistance }(\mathrm{Rgb})\end{array}$ & $\begin{array}{c}\text { Relaxation } \\
\text { time }(\tau)\end{array}$ & $\begin{array}{l}\text { Bulk } \\
\text { resistance } \\
\quad(\mathrm{Rg})\end{array}$ & $\begin{array}{c}\text { Grain } \\
\text { boundary } \\
\text { resistance }(\mathrm{Rgb})\end{array}$ & $\begin{array}{c}\text { Relaxation } \\
\text { time }(\tau)\end{array}$ \\
\hline HAp & $93.9 \mathrm{~K} \Omega$ & - & $3.6 \mathrm{~m} \mathrm{sec}$ & $95.2 \mathrm{~K} \Omega$ & - & $1.0 \mathrm{~m} \mathrm{sec}$ \\
\hline $\begin{array}{l}(0.001 \mathrm{M}) \\
\text { Ag-HAp }\end{array}$ & $69.0 \mathrm{~K} \Omega$ & $253.6 \mathrm{~K} \Omega$ & $1.7 \mathrm{~m} \mathrm{sec}$ & $74.9 \mathrm{~K} \Omega$ & $437.9 \mathrm{~K} \Omega$ & $2.1 \mathrm{~m} \mathrm{sec}$ \\
\hline $\begin{array}{l}(0.005 \mathrm{M}) \\
\text { Ag-HAp }\end{array}$ & $69.01 \mathrm{~K} \Omega$ & $221.7 \mathrm{~K} \Omega$ & $2.0 \mathrm{~m} \mathrm{sec}$ & $87.9 \mathrm{~K} \Omega$ & $609.1 \mathrm{~K} \Omega$ & $2.7 \mathrm{~m} \mathrm{sec}$ \\
\hline $\begin{array}{l}(0.025 \mathrm{M}) \\
\text { Ag-HAp }\end{array}$ & $43.2 \mathrm{~K} \Omega$ & $346.5 \mathrm{~K} \Omega$ & $1.7 \mathrm{~m} \mathrm{sec}$ & $63.9 \mathrm{~K} \Omega$ & $517.3 \mathrm{~K} \Omega$ & $1.3 \mathrm{~m} \mathrm{sec}$ \\
\hline
\end{tabular}


where, $R_{g}$ is grain resistance determined from the intercept of high frequency arc on the real $Z^{\prime}$ axis of impedance spectra and $f_{c}$ is the relaxation frequency corresponding to the maximum value of $Z^{\prime \prime}$. The $C_{g}$ and $C_{g b}$ values calculated for Ca-HAp and Ag-HAp scaffolds, before and after incubation in SBF, are shown in Table 2.

High frequency arcs for all scaffolds before incubation as a function of concentration are depicted in Figure 6(a). The heights of high frequency arcs of impedance spectra do not show any appreciable change for variations in ion concentration excluding scaffold with $0.005 \mathrm{M}$ silver ion content. This scaffold exhibits lowest amplitude with the lowest grain resistance effect.

Cole-Cole plots for SBF incubated Ag-HAp scaffolds as a function of incubation period and silver ion concentration are depicted in Figure 6(b) and Figure $6(c)$. Scaffolds incubated for 8 days do not reveal any perceptible change in amplitude of high frequency arcs. However, incubation for variable time durations show influence on the amplitude of high frequency arcs. The diameter and amplitude of each semi-circle in low frequency region found to be increasing with increase in silver ion concentration (Figure 6(a)). A scaffold with highest silver content results in higher grain boundary resistance. It is also observed that the amplitude and position of low frequency arcs change significantly upon incubation (Figure 6(b) and Figure 6(c)).

For fixed SBF treatment duration, the maximum height for semi-circle is exhibited by Ca-HAp scaffold followed by Ag-HAp scaffold (0.005 M) along with highest grain boundary resistance. The dependence of amplitudes of low frequency arcs and grain boundary resistances on duration of SBF treatment can be revealed from Figure 6(c). The grain boundary resistance decreases with increase in incubation duration from 8 - 32 days for Ag-HAp scaffold (0.005 M). The change in amplitude \& position of low frequency arcs in impedance spectra for incubated scaffolds may be due to the development of calcified tissues on the surface of scaffolds. These changes in scaffolds, after SBF treatment, affect the gain boundary resistance. These findings suggest that the impedance spectroscopy can be useful technique to determine in-vitro bioactivity of scaffolds.

Table 2. Grain capacitance $\left(\mathrm{C}_{\mathrm{g}}\right)$ and grain boundary capacitance $\left(\mathrm{C}_{\mathrm{gb}}\right)$ for Ca-HAp and Ag-HAp scaffolds prior to incubation and after incubation in SBF.

\begin{tabular}{ccccc}
\hline & \multicolumn{2}{c}{ Before incubation in SBF } & After incubation in SBF for 8 days \\
\cline { 2 - 5 } Scaffolds & $\begin{array}{c}\text { Bulk } \\
\text { capacitance } \\
(\mathrm{Cg})\end{array}$ & $\begin{array}{c}\text { Grain boundary } \\
\text { capacitance } \\
(\mathrm{Cgb})\end{array}$ & $\begin{array}{c}\text { Bulk } \\
\text { capacitance } \\
(\mathrm{Cg})\end{array}$ & $\begin{array}{c}\text { Grain boundary } \\
\text { capacitance } \\
(\mathrm{Cgb})\end{array}$ \\
\hline HAp & $38 \mathrm{nf}$ & - & $10 \mathrm{nf}$ & - \\
$(0.001 \mathrm{M})$ Ag-HAp & $24 \mathrm{nf}$ & $76 \mathrm{nf}$ & $28 \mathrm{nf}$ & $44 \mathrm{nf}$ \\
$(0.005 \mathrm{M}) \mathrm{Ag}-\mathrm{HAp}$ & $29 \mathrm{nf}$ & $85 \mathrm{nf}$ & $31 \mathrm{nf}$ & $31 \mathrm{nf}$ \\
$((0.025 \mathrm{M})$ Ag-HAp & $39 \mathrm{nf}$ & $43 \mathrm{nf}$ & $20 \mathrm{nf}$ & $29 \mathrm{nf}$ \\
\hline
\end{tabular}




\subsection{Photoluminescence Analysis}

The photoluminescence spectra of SBF treated Ag-HAp scaffold $(0.005 \mathrm{M})$ as a function of immersion period is shown in Figure 7. From the figure, it can be concluded that all Ag-HAp scaffolds follow similar trend. With an excitation wavelength of $415 \mathrm{~nm}$, the emission spectrum of Ag-HAp, prior to incubation, exhibits a very intense, sharp and narrow blue emission band localized at $\sim 470$ $\mathrm{nm}$. It can also be seen clearly that the relative photoluminescence intensity of this peak changes with change in SBF incubation period. Higher the period of incubation, higher is the intensity. It may be due to increase in thickness of additional biologically active layer formed on the surface upon incubation.

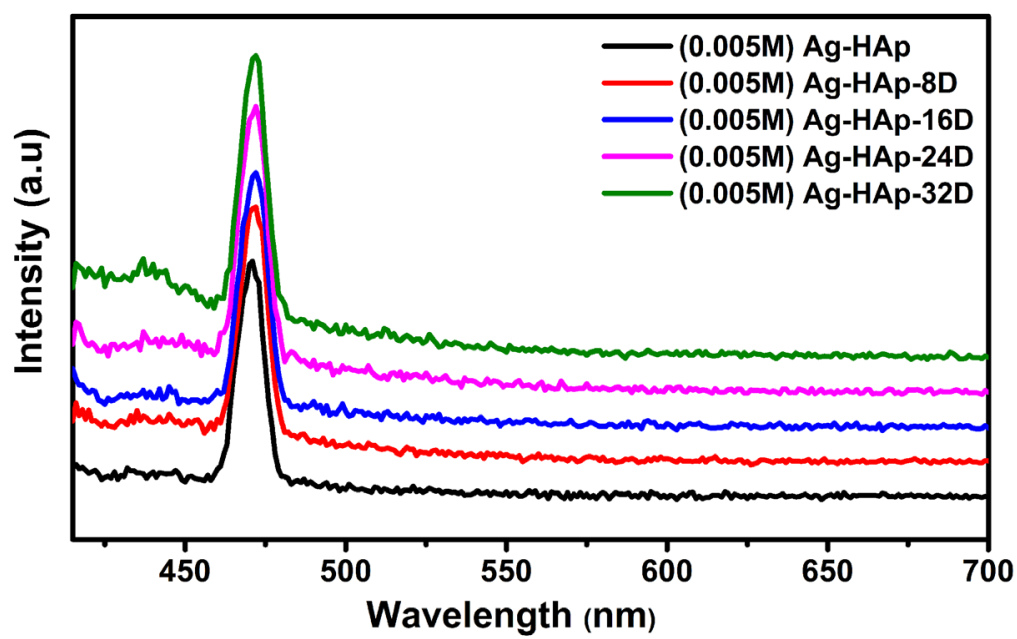

Figure 7. Photoluminescence spectra for Ag-HAp scaffolds after incubation in SBF for various time durations with an excitation wavelength, $\lambda_{\mathrm{exc}}=415 \mathrm{~nm}$ carried out at room temperature.

\section{Conclusions}

Ag-HAp scaffolds can be a potential bioactive biomaterial. The in-vitro bioactivity studies, which reveal development of biological apatite layer on surface of Ag-HAp, are well supported by various characterization techniques like XRD, FTIR, Raman, SEM/EDAX. The XRD profiles of the hydroxyapatite samples, before and after incubation, clearly demonstrate that partial replacement of calcium ions by silver ions does not show any appreciable effect on hexagonal phase and structure remains intact during the incubation process.

Dielectric parameters are found to be dependent on concentration of silver ions and period of incubation. The changes in dielectric constant and dissipation factor can be attributed to accumulation of phosphate and calcium ions layer on the surface of HAp and Ag-HAp scaffolds upon SBF treatment. The changes in amplitude and position of low $\&$ high frequency branches in Cole-Cole plots, upon incubation \& with ion content, indicate formation of apatite layer on scaffold surface. Also, Ag-HAp scaffolds, with and without incubation in SBF, show excellent optical performance. 


\section{Conflicts of Interest}

The authors declare no conflicts of interest regarding the publication of this paper.

\section{References}

[1] Mondal, S., Pal, U. and Dey, A. (2016) Natural Origin Hydroxyapatite Scaffold as Potential Bone Tissue Engineering Substitute. Ceramic International, 42, 18338-18346. https://doi.org/10.1016/j.ceramint.2016.08.165

[2] Pon-On, W., Suntornsaratoon, P., Charoenphandhu, N., Thongbunchoo, J., Karishnamra, N. and Tang, I.M. (2016) hydroxyapatite from Fish Scale for Potential Use as Bone Scaffold or Regenerative Material. Material Science and Engineering $C$, 62, 183-189. https://doi.org/10.1016/j.msec.2016.01.051

[3] Mondal, S., Hoang, G., Manivasagan, P., Moorthy, M.S., Kim, H.H., Phan, T.T.V. and Oh, J. (2019) Comparative Characterization of Biogenic and Chemical Synthesized Hydroxyapatite Biomaterials for Potential Biomedical Application. Material Chemistry and Physics, 228, 344-356. https://doi.org/10.1016/j.matchemphys.2019.02.021

[4] Zhang, K., Zeng, K., Shen, C., Tian, S. and Yang, M. (2018) Determination of Protein Kinase A Activity and Inhibition by Using Hydroxyapatite Nanoparticles as a Fluorescent Probe. Mikrochimica Acta, 185, 225. https://doi.org/10.1007/s00604-018-2754-1

[5] Verma, A.H., Sampath Kumar, T.S., Madhumathi, K., Rubaiya, Y., Ramalingan, M. and Doble, M. (2019) Curcumin Releasing Eggshell Derived Carbonated Apatite Nanocarriers for Combined Anti-Cancer, Anti-Inflammatory and Bone Regenerative Therapy. Journal of Nanoscience and Nanotechnology, 19, 6872-6880. https://doi.org/10.1166/jnn.2019.16640

[6] Zhao, L., Zhao, W., Liu, Y., Chen, X. and Wang, Y. (2017) Nano-Hydroxyapatite-Derived Drug and Gene Co-Delivery System for Anti-Angiogenesis Therapy of Breast Cancer. Medical Science Monitor, 23, 4723-4732. https://doi.org/10.12659/MSM.902538

[7] Li, M., Xiong, P., Yan, F., Li, S., Ren, C., Yin, Z., Li, A., Li, H., Ji, X., Zheng, Y. and Cheng, Y. (2018) An Overview of Graphene-Based Hydroxyapatite Composites for Orthopaedic Applications. Bioactive Materials, 3, 1-18.

https://doi.org/10.1016/j.bioactmat.2018.01.001

[8] Yu, W., Sun, T.W., Ding, Z., Qi, C., Zhao, H., Chen, F., Shi, Z., Zhu, Y.J., Chen, D. and He, Y. (2017) Copper-Doped Mesoporous Hydroxyapatite Microspheres Synthesized by a Microwave-Hydrothermal Method Using Creatine Phosphate as an Organic Phosphorus Source: Application in Drug Delivery and Enhanced Bone Regeneration. Journal of Materials Chemistry B, 5, 1039-1052. https://doi.org/10.1039/C6TB02747D

[9] Mahabole, M., Bahir, M. and Khairnar, R. (2013) Mn Blended Hydroxyapatite Nanoceramic: Bioactivity, Dielectric and Luminescence Studies. Journal of Biomimetics, Biomaterials and Tissue Engineering, 18, 43-59. https://doi.org/10.4028/www.scientific.net/JBBTE.18.43

[10] Kim, B., Yang, S., Yoon, J. and Lee, J. (2017) Enhanced Bone Regeneration by Silicon-Substituted Hydroxyapatite Derived from Cuttlefish Bone. Clinical Oral Implants Research, 28, 49-56. https://doi.org/10.1111/clr.12613

[11] Tsai, S.W., Yu, W.X., Hwang, P.A., Huang, S.S., Lin, H.M., Hsu, Y.W. and Hsu, F.Y. (2018) Fabrication and Characterization of Strontium-Substituted Hydroxyapa- 
tite- $\mathrm{CaO}-\mathrm{CaCO}_{3}$ Nanofibers with a Mesoporous Structure as Drug Delivery Carriers. Pharmaceutics, 10, 179. https://doi.org/10.3390/pharmaceutics10040179

[12] Frasnelli, M., Cristofaro, F., Sglavo, V.M., Dire, S., Callonea, E., Ceccato, R., Bruni, G., Cornaglia, A.I. and Visai, L. (2017) Synthesis and Characterization of Strontium-Substituted Hydroxyapatitenanoparticles for Bone Regeneration. Materials Science and Engineering C, 71, 653-662. https://doi.org/10.1016/j.msec.2016.10.047

[13] Kargozar, S., Lotfibakhshaiesh, N., Ai, J., Mozafari, M., Milan, P.B., Hamzehlou, S., Barati, M., Baino, F., Hill, R.G. and Joghataei, M.T. (2017) Strontium- and Cobalt-Substituted Bioactive Glasses Seeded with Human Umbilical Cord Perivascular Cells to Promote Bone Regeneration via Enhanced Osteogenic and Angiogenic Activities. Acta Biomaterialia, 58, 502-514. https://doi.org/10.1016/j.actbio.2017.06.021

[14] Yu, W., Sun, T.W., Qi, C., Ding, Z., Zhao, H., Zhao, S., Shi, Z., Zhu, Y.J., Chen, D. and He, Y. (2017) Evaluation of Zinc-Doped Mesoporous Hydroxyapatite Microspheres for the Construction of a Novel Biomimetic Scaffold Optimized for Bone Augmentation. International Journal of Nanomedicine, 12, 2293-2306. https://doi.org/10.2147/IJN.S126505

[15] Qiao, H., Song, G., Huang, Y., Yang, H., Han, S., Zhang, X., Wang, Z., Ma, J., Bu, X. and Fu, L. (2019) Si, Sr, Ag Co-Doped Hydroxyapatite/TiO ${ }_{2}$ Coating: Enhancement of Its Antibacterial Activity and Osteoinductivity. RSC Advances, 9, 13348-13364. https://doi.org/10.1039/C9RA01168D

[16] Rodriguez, D.A.L., de Lima, R., Fraceto, L.F., Perez, A.L., Dominguez, M.B., Batres, R.G., Rojas, A.R. and Carmona, V.O. (2017) Development of HA/Ag-NPs Composite Coating from Green Process for Hip Applications. Molecules, 22, 1291. https://doi.org/10.3390/molecules22081291

[17] Akiyama, T., Miyamoto, H., Yonekura, Y., Tsukamoto, M., Ando, Y., Noda, I., Sonohata, M. and Mawatari, M. (2013) Silver Oxide-Containing Hydroxyapatite Coating Has in Vivo Antibacterial Activity in the Rat Tibia. Journal of Orthopaedic Research, 31, 1195-1200. https://doi.org/10.1002/jor.22357

[18] Fonseca, F.M., Costa, A.M., Campos, J.B., LSB Marcal, R., da Rocha, D.N. and da Silva, M.H. (2017) Bioactivity Assessment of Ag-HA. Biomaterials and Medical Applications, 1, 2. https://doi.org/10.4172/2577-0268.1000106

[19] Yan, Y., Zhang, X., Huang, Y., Ding, Q. and Pang, X. (2014) Antibacterial and Bioactivity of Silver Substituted Hydroxyapatite/ $/ \mathrm{TiO}_{2}$ Nanotube Composite Coatings on Titanium. Applied Surface Science, 314, 348-357.

https://doi.org/10.1016/j.apsusc.2014.07.027

[20] Erakovic, S., Jankovic, A., Veljovic, D., Palcevskis, E., Mitric, M., Stevanovic, T., Janackovic, D. and Stankovic, V.M. (2013) Corrosion Stability and Bioactivity in Simulated Body Fluid of Silver/Hydroxyapatite and Silver/Hydroxyapatite/Lignin Coatings on Titanium Obtained by Electrophoretic Deposition. The Journal of Physical Chemistry B, 117, 1633-1643. https://doi.org/10.1021/jp305252a

[21] Sivolella, S., Stellini, E., Brunello, G., Gardin, C., Ferroni, L., Bressan, E. and Zavan, B. (2012) Silver Nanoparticles in Alveolar Bone Surgery Devices. Journal of Nanomaterials, 2012, Article ID: 975842. https://doi.org/10.1155/2012/975842

[22] Diago, P., S. Bielsa, J.M. and G. Escoda, C. (2001) Periapical Surgery of 31 Lower Molars Based on the Ultrasound Technique and Retrograde Filling with Silver Amalgam. Medicina Oral, Patología Oral y Cirugía Bucal, 6, 376-382.

[23] Sierpowska, J., Toyras, J., Hakulinen, M.A., Saarakkala, S., Jurvelin, J.S. and Lappalainen, R. (2003) Electrical and Dielectric Properties of Bovine Trabecular Bone-Relationships with Mechanical Properties and Mineral Density. Physics in 
Medicine \& Biology, 48, 775-786. https://doi.org/10.1088/0031-9155/48/6/306

[24] Balmer, T.W., Vesztergom, S., Broekmann, P., Staheland, A. and Buchler, P. (2018) Characterization of the Electrical Conductivity of Bone and Its Correlation to Osseous Structure. Scientific Reports, 8, 8601. ttps://doi.org/10.1038/s41598-018-26836-0

[25] Petrov, I., Kalinkevich, O., Pogorielov, M., Kalinkevich, A., Stanislavov, A., Sklyar, A., Danilchenko, S. and Yovcheva, T. (2016) Dielectric and Electric Properties of New Chitosan-Hydroxyapatite Materials for Biomedical Application: Dielectric Spectroscopy, and Corona Treatment. Carbohydrate Polymers, 151, 770-778. https://doi.org/10.1016/j.carbpol.2016.05.110

[26] Shayesteh, Y.S., Eslami, B., Dehghan, M.M., Vaziri, H., Alikhassi, M., Mangoli, A. and Khojasteh, A. (2007) The Effect of a Constant Electrical Field on Osseointegration after Immediate Implantation in Dog Mandibles: A Preliminary Study. Journal of Prosthodontics, 16, 337-342. https://doi.org/10.1111/j.1532-849X.2007.00208.x

[27] Nakamura, S., Kobayashi, T., Nakamura, M., Itoh, S. and Yamashita, K. (2010) Electrostatic Surface Charge Acceleration of Bone Ingrowth of Porous Hydroxyapatite/ $\beta$-Tricalcium Phosphate Ceramics. Journal of Biomedical Materials Research Part A, 92A, 267-275. https://doi.org/10.1002/jbm.a.32354

[28] Huegel, J., Choi, D.S. and Nuss, C.A. (2018) Effects of Pulsed Electromagnetic Field Therapy at Different Frequencies and Durations on Rotator Cuff Tendon-to-Bone Healing in a Rat Model. Journal of Shoulder and Elbow Surgery, 27, 553-560. https://doi.org/10.1016/j.jse.2017.09.024

[29] Midura, R.J., Ibiwoye, M.O. and Powell, K.A. (2005) Pulsed Electromagnetic Field Treatments Enhance the Healing of Fibular Osteotomies. Journal of Orthopaedic Research, 23, 1035-1046. https://doi.org/10.1016/j.orthres.2005.03.015

[30] Lin, M.C., Yang, F., Herfat, S.T., Bahney, C.S., Marmor, M. and Maharbiz, M.M. (2017) New Opportunities for Fracture Healing Detection: Impedance Spectroscopy Measurements Correlate to Tissue Composition in Fractures. Journal of Orthopaedic Research, 35, 2620-2629. https://doi.org/10.1002/jor.23570

[31] Kozhevnikov, E., Hou, X., Qiao, S., Zhao, Y., Li, C. and Tian, W. (2016) Electrical Impedance Spectroscopy-a Potential Method for the Study and Monitoring of a Bone Critical-Size Defect Healing Process Treated with Bone Tissue Engineering and Regenerative Medicine Approaches. Journal of Materials Chemistry B, 4, 2757-2767. https://doi.org/10.1039/C5TB02707A

[32] Chavan, P.N., Bahir, M.M., Mene, R.U., Mahabole, M.P. and Khairnar, R.S. (2010) Study of Nanobiomaterial Hydroxyapatite in Simulated Body Fluid: Formation and Growth of Apatite. Materials Science and Engineering B, 168, 224-230. https://doi.org/10.1016/j.mseb.2009.11.012

[33] Mahabole, M.P., Bahir, M.M., Kalyankar, N.V. and Khairnar, R.S. (2012) Effect of Incubation in Simulated Body Fluid on Dielectric and Photoluminescence Properties of Nano-Hydroxyapatite Ceramic Doped with Strontium Ions. Journal of Biomedical Science and Engineering, 5, 396-405. https://doi.org/10.4236/jbise.2012.57050

[34] Jalota, S., Bhaduri, S.B. and Tas, A.C. (2008) Using a Synthetic Body Fluid (SBF) Solution of $27 \mathrm{mM} \mathrm{HCO}_{3}^{-}$to Make Bone Substitutes More Osteointegrative. Material Science and Engineering C, 28, 129-140. https://doi.org/10.1016/j.msec.2007.10.058

[35] Antonakos, A., Liarokapis, E. and Leventouri, T. (2007) Micro-Raman and FTIR Studies of Synthetic and Natural Apatites. Biomaterials, 28, 3043-3054. 
https://doi.org/10.1016/j.biomaterials.2007.02.028

[36] Liu, L., Wu, Y., Xu, C., Yu, S., Wu, X. and Dai, H. (2018) Synthesis, Characterization of Nano- $\beta$-Tricalcium Phosphate and the Inhibition on Hepatocellular Carcinoma Cells. Journal of Nanomaterials, 2018, Article ID: 6468246.

https://doi.org/10.1155/2018/6468246 